\title{
Theory of Gain, Modulation Response, and Spectral Linewidth in AlGaAs Quantum Well Lasers
}

\author{
YASUHIKO ARAKAWA, MEMBER, IEEE, AND AMNON YARIV, FELLOW, IEEE
}

\begin{abstract}
We investigate theoretically a number of important issues related to the performance of AlGaAs quantum well (QW) semiconductor lasers. These include a basic derivation of the laser gain, the linewidth enhancement factor $\alpha$, and the differential gain constant in single and multiple QW structures. The results reveal the existence of gain saturation with current in structures with a small number of wells. They also point to a possible two-fold increase in modulation bandwidth and a ten-fold decrease in the spectral laser linewidth in a thin $Q W$ laser compared to a conventional double heterostructure laser.
\end{abstract}

\section{INTRODUCTION}

$\mathrm{T}$ HE application of semiconductor lasers for optical communication requires low-noise characteristics and high-frequency dynamic performance. These properties have received considerable attention in conventional double heterostructure (DH) lasers [1]-[5]. Recently the detuned loading by the use of coupled-cavity DH lasers was proposed for improving these properties [6]. In this structure, the differential gain $g^{\prime}$ and the linewidth enhancement factor $\alpha$ which determine, respectively, the dynamic performance and noise characteristics, are varied by detuning the lasing frequency away from the loss peak. The basic quantum mechanical expressions for $g^{\prime}$ and $\alpha$ suggest yet another way to improve $\alpha$ and $g^{\prime}$ through changes in the density of states which occur in quantum well (QW) structures. Although QW lasers have proven to be attractive light sources because of their low threshold current densities and the low temperature sensitivity of the threshold current [7]-[11], little effort has been directed towards measurement or calculation of these noise characteristics and high-modulation performance [12]-[14].

In this paper, we investigate theoretically the effects of QW structure parameters such as the number and the thickness of QW's on the dynamic response and quantum noise, and consider the optimum QW structure for realizing low-noise characteristics and/or high-dynamic performance. In the analysis, we also consider the important issue of the use of multiquantum well structures in order to avoid the phenomenon of gain saturation with current, as well as for improving the noise and dynamic performance. The effect of the structural parameters such as the thickness and the number of QW's on the threshold cur-

Manuscript received February 8, 1985; revised May 13, 1985. This work was supported by the Air Force Office of Scientific Research, the Office of Naval Research, the ITT Corporation, and the Japan Society for Promotion of Science.

The authors are with the California Institute of Technology, Pasadena, CA 91125 . rent have been considered before [15], [16], although the gain saturation effect was not explicitly treated. In Section III the fundamental properties of differential gain are discussed, and then the effects of QW structure parameter on the laser dynamics are investigated. In Section IV, the linewidth enhancement factor $\alpha$ and the spontaneous emission factor are studied and the attainable reduction of the linewidth by the use of the optimum QW structures is considered. The results indicate that the modulation bandwidth (relaxation resonance frequency) is enhanced by a factor of $\sim 2$ in the multiquantum well (MQW) laser and the laser linewidth (FM noise) is found to be significantly suppressed, by $\sim \frac{1}{10}$, in a single thin QW (SQW) laser compared to a conventional double heterostructure laser. In order to clarify achievable limits of these properties with quantum well effects, we will consider AlGaAs quantum well lasers which are almost free from nonradiative processes, such as the Auger recombination at room temperature.

\section{Linear Gain and Threshold Current}

\section{A. Density of States}

In the QW structures [7], a series of energy levels and associated subbands are formed owing to the quantization of electrons in the direction of the quantum well thickness. The density of states (per unit energy per unit area) of such confined electrons in the $n$th subband is given by

$$
\rho_{c_{n}}(E)=\frac{m_{c}}{\pi \hbar^{2}} H\left(E-\epsilon_{c_{n}}\right)
$$

where $H(x), m_{c}, \hbar, L_{z}$, and $\epsilon_{c_{n}}$ are the Heaviside function, the effective mass of electrons, Planck's constant $(h)$ divided by $2 \pi$, the QW thickness, and the quantized level of electrons in the $n$th subband of the QW structures, respectively. When the barrier height is sufficiently big and the barrier thickness is sufficiently large, $\epsilon_{c_{n}}$ is equal to $(n \pi \hbar)^{2} /\left(2 m_{c} L_{z}^{2}\right)$. On the other hand, if the barrier is thin or its barrier height is small so that coupling between adjacent wells exists, the degeneracy of the individual well quantized energy levels dissappears and each single-well level splits into $N$ different energy levels. In order to simplify the discussion in this paper, we will consider the weak coupling limit of QW structures in which energy broadening due to coupling is sufficiently small compared to the energy broadening (usually more than $5 \mathrm{meV}$ ) due to intraband relaxation time. 


\section{B. Linear Gain and Threshold Current}

When the recombination is dominated by band-to-band radiative process, the linear bulk gain derived under $k$-selection rule is given by

$$
\begin{aligned}
g(E)= & \int A(\epsilon) \sum_{j=h, l} \sum_{n=1}^{\infty} \frac{\rho_{\text {red }}^{j}(\epsilon)}{L_{z}}\left(f_{c}\left(E_{c_{n}}\right)\right. \\
& \left.-f_{v}\left(E_{v_{n}}^{j}\right)\right) \frac{\left(\hbar / \tau_{i n}\right)}{(E-\epsilon)^{2}+\left(\hbar / \tau_{\text {in }}\right)^{2}} d \epsilon .
\end{aligned}
$$

The bulk gain is the gain exercised by an electromagnetic field if it were completely confined to the quantum well. $E$ is the photon energy, $j$ designates either light holes (1) or heavy holes $(h), \rho_{\text {red }}^{j}$ is the reduced density of states which is defined by $\rho_{\text {redn }}^{j}=\left(\left(\rho_{c_{n}}\right)^{-1}+\left(\rho_{v_{n}}^{i}\right)^{-1}\right)^{-1}, \rho_{v_{n}}^{j}$ is the density of states of light holes $(j=1)$ or heavy holes $(j=h), f_{c}\left(f_{v}\right)$ is the Fermi-Dirac distribution function for electrons (holes) in the conduction band (the valence band) with the Fermi-energy $\epsilon_{F_{c}}\left(\epsilon_{F_{v}}\right), \tau_{\text {in }}$ is the intraband relaxation time. $E_{c_{n}}$ and $E_{v_{n}}^{j}$ are equal to $\left(m_{c} \epsilon_{v_{\eta}}^{j}+\right.$ $\left.m_{v}^{j} E+m_{v}^{j} \epsilon_{c_{n}}\right) /\left(m_{c}+m_{v}^{j}\right)$, and $\left(m_{c} \epsilon_{v_{n}}^{j}-m_{c} E+m_{v}^{j} \epsilon_{c_{n}}\right) /$ $\left(m_{c}+m_{v}^{j}\right)$, respectively, where $m_{v}^{j}$ and $\epsilon_{v_{n}}^{j}$ are the effective mass and the energy level of the $n$th subband of heavy holes $(j=h)$ or light holes $(j=1) . A(\epsilon)$ is the coefficient related to the square of the dipole matrix element $|M|_{\text {ave }}^{2}$ as given by

$$
A(\epsilon)=\frac{\pi e^{2} h}{m_{0}^{2} c n_{r} E_{g}}|M|_{\text {ave }}^{2}
$$

where $n_{r}$ is the refractive index of GaAs, $e$ is the electron charge, $m_{0}$ is the mass of electrons, $c$ is the light velocity, $E_{g}$ is the band-gap, and $h$ is the Planck's constant. In quantum well structures, the gain depends on the polarization of the light. The dipole matrix element $|M|_{\text {ave }}^{2}$ which is different from that of the double heterostructure was discussed by Asada et al. [16]. For instance the dipole matrix element for TE mode due to an electron-heavy hole transition is given by

$$
|M|_{\text {ave }}^{2 \mathrm{TE}}=\left|M_{0}\right|_{\text {ave }}^{2}\left(1+E /\left(\epsilon_{c_{n}}-\epsilon_{v_{n}}^{h}\right)\right)
$$

where $\left|M_{0}\right|_{\text {ave }}^{2}$ is the square of the dipole matrix with the conventional double heterostructure lasers and is approximately equal to $1.33 m_{0} E_{g}$. The quasi-Fermi energy $\epsilon_{F_{c}}$ and $\epsilon_{F_{v}}$ are determined by both the charge neutrality condition and the condition that the modal gain $g_{\text {mod }}(E)=\Gamma g(E)$ at the photon energy $E_{1}$ for laser oscillation is equal to the total losses $\alpha_{\text {total }}$ as follows:

$$
\begin{aligned}
g_{\text {mod }}\left(E_{l}\right)=\Gamma g\left(E_{l}\right)=\alpha_{\text {total }} & \\
& \equiv \Gamma \alpha_{a c}+(1-\Gamma) \alpha_{\text {ex }}+L^{-1} \ln (1 / R)
\end{aligned}
$$

where $\alpha_{\mathrm{ac}}, \alpha_{\mathrm{ex}}, R$, and $L$ are the loss in the active region, the loss in the cladding layer, the reflectivity, and the cavity length. The optical confinement factor $\Gamma$ depends on the structure strongly. In this paper, we assume a multiquantum well (MQW) structure composed of thin $\mathrm{GaAs}$ well layers, $\mathrm{Ga}_{0.75} \mathrm{Al}_{0.25} \mathrm{As}$ barrier layers, and $\mathrm{Ga}_{0.75} \mathrm{Al}_{0.25} \mathrm{As}$ waveguide layers. The dimension of the

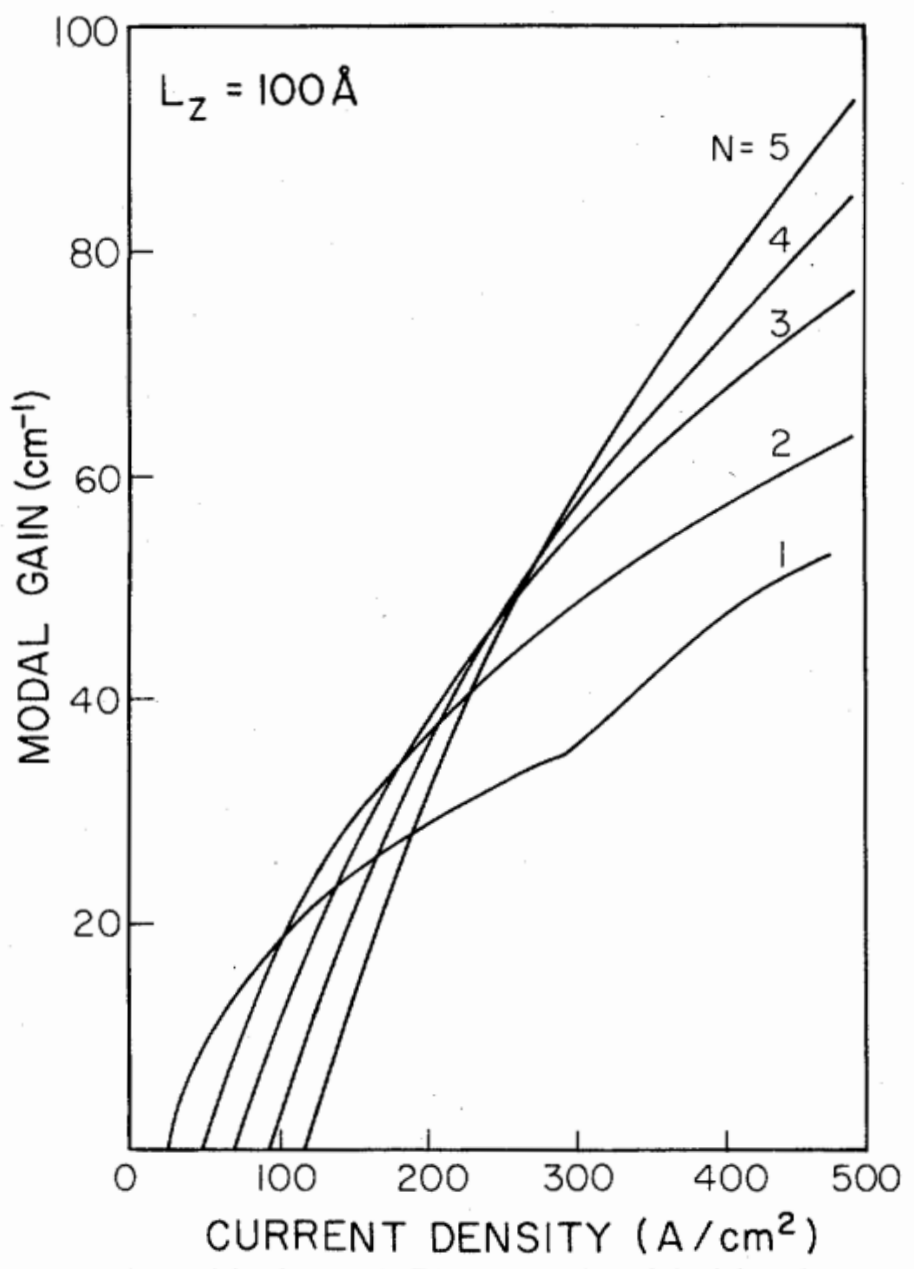

Fig. 1. The modal gain $g_{\text {mod }}\left(=\Gamma_{g}\right)$ as a function of the injected current density $J$ with the various number of quantum wells $N$. In this case, the quantum well thickness $L_{z}$ is assumed to be $100 \AA$.

waveguide layers is determined so that the total thickness, including QW wells, barrier layers, and waveguide layers, is equal to $2000 \AA$. The cladding layers are a $\mathrm{p}-\mathrm{Ga}_{0.6} \mathrm{Al}_{0.4} \mathrm{As}$ layer, and $\mathrm{n}-\mathrm{Ga}_{0.6} \mathrm{Al}_{0.4} \mathrm{As}$ layer, respectively. In particular, when the number of $\mathrm{QW}$ is equal to 1 , we call this structure a single QW (SQW) structure, hereafter. In these MQW structures the $\Gamma$ is given approximately by [15]

$$
\Gamma=0.3 N\left(L_{z} / L_{0}\right)
$$

where $L_{0}$ is equal to $1000 \AA, N$ is the number of QW's.

Fig. 1 shows the maximum gain $g_{\bmod }\left(E_{l}\right)$ exercised by the laser mode as a function of the injected current density $J$ for different number of QW's. The injected current $J$ is given by

$$
\begin{gathered}
J=e L_{z} N\left(\iint \frac{8 \pi n_{r}^{2} E_{g}^{2}}{c^{2} h} A(\epsilon) \sum_{j=h, l} \sum_{n=1}^{\infty} \frac{\rho_{\mathrm{red}_{n}}^{j}(\epsilon)}{L_{z}} f_{c}\left(E_{c_{n}}\right)\right. \\
\left.\cdot\left(1-f_{v}\left(E_{v_{n}}^{j}\right)\right) \frac{\left(\hbar / \tau_{\text {in }}\right)}{(E-\epsilon)^{2}+\left(\hbar / \tau_{\text {in }}\right)^{2}} d \epsilon d E\right) .
\end{gathered}
$$

The term surrounded by parenthesis is the total emission rate. In this calculation, the QW thickness $L_{z}$ and $\tau_{\text {in }}$ are 
assumed to be $100 \AA$ and 0.2 ps. The energy band-gap discontinuity $\Delta E_{c}$ and $\Delta E_{v}$ of the conduction band and the valence band at the interface of $\mathrm{GaAs}$ and $\mathrm{Al}_{0.3} \mathrm{Ga}_{0.7} \mathrm{As}$ are set $0.8 \Delta E$ and $0.2 \Delta E$, respectively, where $\Delta E$, which is the total band discontinuity, is assumed to be $333 \mathrm{meV}$. We notice a very marked flattening ("saturation") of the gain at high injected currents, especially in a single well $(N=1)$. This gain saturation is due to the step-like shape of the density of state functions, and the fact that penetration of the quasi-Fermi levels into the conduction band or valence band takes place at high current injection. The product $\rho_{\text {red }}(\epsilon)\left[f_{c}\left(E_{c}\right)-f_{v}\left(E_{v}\right)\right]$, which determines the gain, becomes a constant and no longer increases with current. The maximum gain $g_{\bmod }\left(E_{l}\right)$ available with $N$ QW's is thus $N$ times larger than $g_{\text {mod }}\left(E_{l}\right)$ with $N=1$ since each well can now provide its saturation gain, which is equal to that of a SQW laser. We can consequently avoid the saturation effect by increasing the number of QW's although the injected current to achieve this maximum gain also increases by $N$ times. Owing to this gain saturation effect there exists an optimum number of QW's for minimizing the threshold current for a given total loss $\alpha_{\text {total }}$. From Fig. 1 we see that, for low losses, the injected threshold current is minmimum in the case of $N=1$. On the other hand, if the $\alpha_{\text {total }}>20 \mathrm{~cm}^{-1}$, the threshold current with $N=1$ is larger than that of $N=2$. At higher values of $\alpha_{\text {total }}$, which call for large laser modal gain $g_{\text {mod }}$, a larger number of wells are needed. From Fig. 1 we see that for $\alpha_{\text {loss }}>50 \mathrm{~cm}^{-1}$ a five-well structure $(N=5)$ will have the lowest threshold current.

In order to clarify these situations, in Fig. 2 the threshold current as a function of the QW number with various total loss is shown. This figure indicates that when the $\alpha_{\text {total }}$ is low, $N=1$ is optimum, whereas the optimum number $N$ is larger than 1 for higher $\alpha_{\text {loss. }}$. Thus the gain saturation which is enhanced in a SQW structure causes the significant dependence of the optimum number $N$ on the $\alpha_{\text {total }}$ for reducing threshold current.

There remains another important structural parameter, the quantum well thickness. Fig. 3 shows the threshold current change as a function of the QW thickness. In this calculation, the number of QW's with each QW thickness is optimized so that the threshold current is minimum. The results indicate that the threshold current of thinner QW lasers $\left(L_{z}=50 \sim 100 \AA\right)$ is much lower than that of thicker QW lasers. We also notice that the threshold current is minimized with $L_{z} \sim 60 \AA$ when $\alpha_{\text {loss }}$ is low $\left(\alpha_{\text {loss }}=10,30 \mathrm{~cm}^{-1}\right)$. This is mainly due to the fact that the current for transparency (gain is just equal to zero), which corresponds approximately to threshold current with extremely low loss, is minimized at the thickness of $L_{z} \sim$ $60 \AA$ in the case of $N=1$ and also due to the fact that the optimum $N$ at each QW is 1 in the case of low $\alpha_{\text {loss }}$ for thin quantum well structures. Since the gain saturation is more enhanced for smaller $L_{z}$, the optimum $N$ of thinner QW's is larger than that of thicker QW's. For $\alpha_{\text {loss }}=50$ $\mathrm{cm}^{-1}, N_{\text {opt }}\left(L_{z}=50 \AA\right)=4$, while $N_{\text {opt }}\left(L_{z}=100 \AA\right)=$ 2 and $N_{\text {opt }}\left(L_{z}=200 \AA\right)=1$.

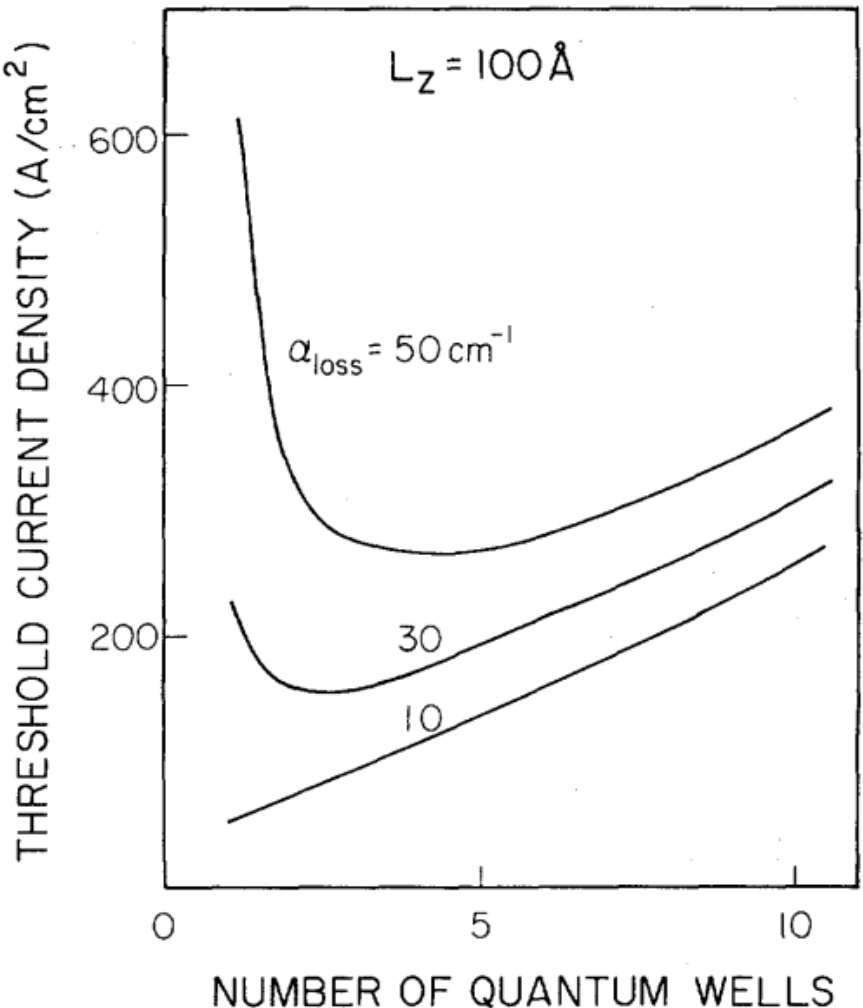

Fig. 2. The threshold current change as a function of the number $N$ of quantum wells with the various total loss $\alpha_{\text {loss }}$. In this case, the quantum well thickness $L_{z}$ is assumed to be $100 \AA$.

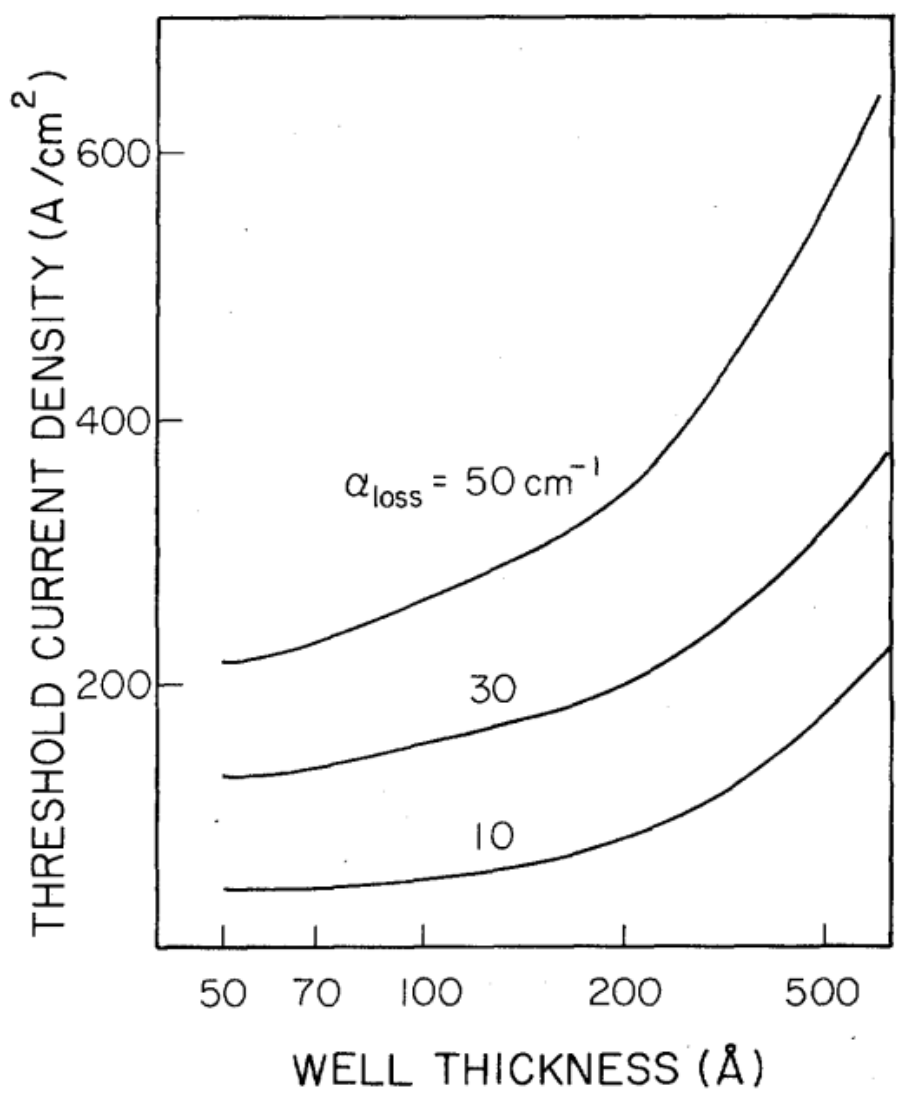

Fig. 3. The threshold current change as a function of the quantum well thickness $L_{z}$ with various total loss $\alpha_{\text {loss }}$. The number $N$ of quantum wells which is optimized so that threshold current is minimum. 


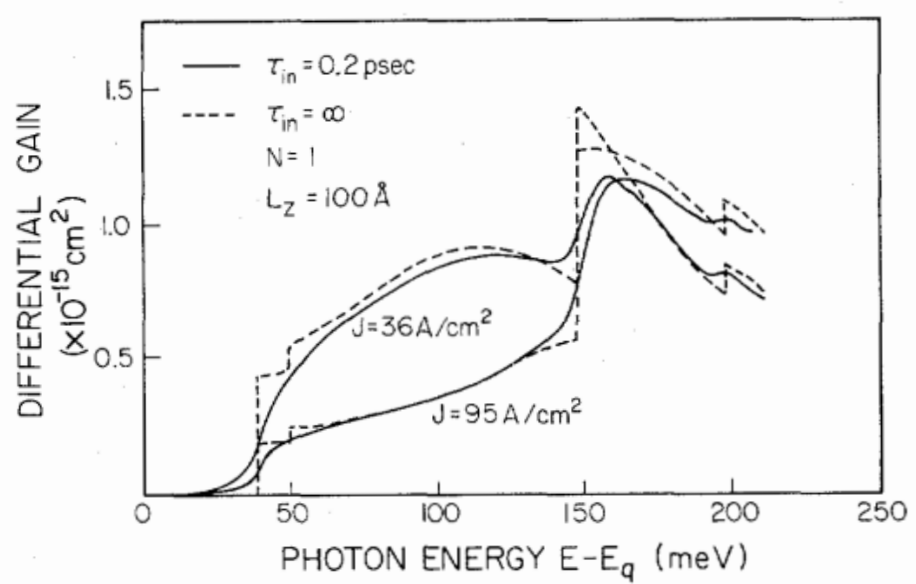

Fig. 4. The differential gain $g^{\prime}(E)$ with several injected currents as a function of the photon energy substracted the band-gap energy of GaAs $\left(E-E_{g}\right)$. In this calculation $\tau_{\text {in }}$ is assumed to be $0.2 \mathrm{ps}$ (solid curve) and $\infty \mathrm{s}$ (broken curve). The number of the quantum wells is 1 and the thickness is $100 \AA$.

\section{Differential Gain and Modulation Bandwidth}

\section{A. Differential Gain}

In this section, we investigate the properties of the differential gain and calculate the modulation bandwidth of the quantum well laser. The differential gain $g^{\prime}(n, E)$ at a photon energy $E$ is defined as the derivative of the bulk gain with respect to carrier concentration, i.e., $\left(g^{\prime}(n\right.$, $E) \equiv d g(n, E) / d n)$. Since it figures in the modulation response of the semiconductor laser through the expression $f_{r}=\frac{1}{2} \pi \sqrt{g^{\prime}(n, E)\left(n_{r} / c\right)\left(P_{0} / \tau_{p}\right)}$ (where $f_{r}$ is the relaxation frequency conventionally used as a measure of the upper useful modulation frequency [1], $P_{0}$ is the photon density in the active region and $\tau_{p}$ is the photon lifetime in the passive cavity), $g^{\prime}(n, E)$ contains the "physics" of the semiconductor laser. The derivation of $f_{r}$ will be discussed in the next section. In order to emphasize the carrier dependence of the gain, we describe the gain as a function of both $E$ and $n$. When we take broadening effects due to intraband relaxation into consideration the differential gain is given by

$$
\begin{aligned}
g^{\prime}(n, E) \frac{d g(n, E)}{d n}=\int A(\epsilon) \sum_{j=h, l} \sum_{n=1}^{\infty} \frac{\rho_{\text {red }_{n}}^{j}(\epsilon)}{L_{z}} \\
\cdot\left(\frac{d f_{c}\left(\epsilon_{c_{n}}\right)}{d n}-\frac{d f_{v}\left(\epsilon_{v_{n}}^{j}\right)}{d n}\right) \cdot \frac{\left(\hbar / \tau_{\text {in }}\right)}{(E-\epsilon)^{2}+\left(\hbar / \tau_{\text {in }}\right)^{2}} d \epsilon .
\end{aligned}
$$

Fig. 4 shows the differential gain $g^{\prime}(n, E)$ as a function of the excess photon energy $\left(E-E_{g}\right)\left(E_{g}=\right.$ band-gap energy) at two injected current densities $(J=36$, $95 \AA / \mathrm{cm}^{-2}$ ) in SQW laser with a QW thickness of $L_{z}=$ $100 \mathrm{~A}$. The solid curve is that of $g^{\prime}(n, E)$ with $\tau_{\text {in }}=0.2$ ps and the broken line represents $g^{\prime}(n, E)$ with $\tau_{\text {in }}=\infty$ (i.e., no energy broadening). We call $g^{\prime}(n, E)$ without energy broadening the differential gain profile, hereafter. This differential gain profile is 0 below $39 \mathrm{meV}$ which corresponds to the energy difference between the first subband of electrons and heavy holes $\left(\epsilon_{c_{1}}-\epsilon_{\mathrm{v}_{1}}^{h}-E_{g}\right)$. The

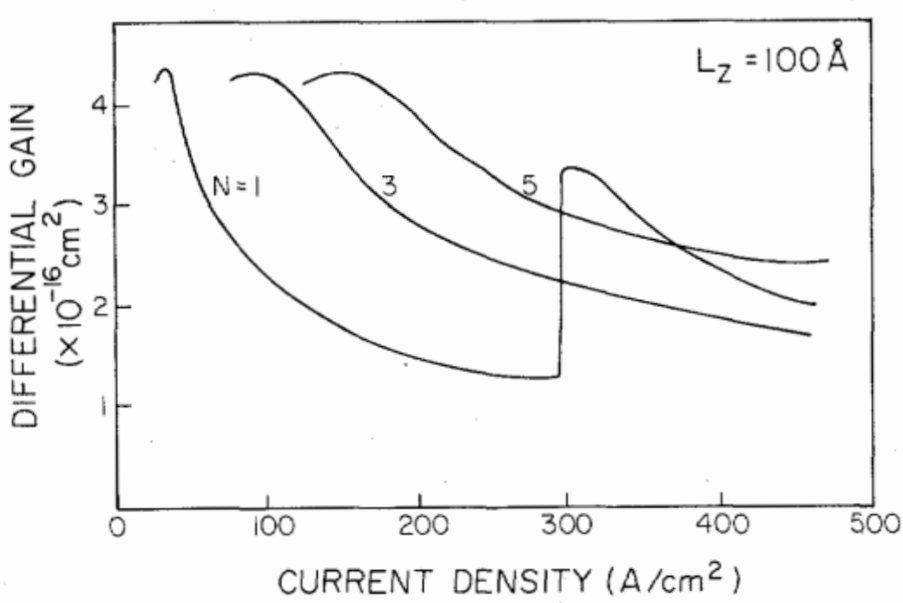

Fig. 5. The differential gain $g^{\prime}\left(n, E_{l}\right)$, at which the gain is maximum, is shown as a function of the injected current $J$ with various number of quantum wells. The thickness of quantum wells is $100 \AA$.

abrupt small change near $50 \mathrm{meV}$ is due to the onset of the electron-light hole transition of the first subband. We also note other abrupt changes in the $g^{\prime}(E, n)$ near 150 $\mathrm{meV}$ and $190 \mathrm{meV}$. These changes correspond to the electron-heavy hole, and electron-light hole transitions of the second subband, respectively. In the case of the more realistic $\tau_{\text {in }}=0.2$ ps curves, the energy broadening of the abrupt features, by $\left(\hbar / \tau_{\text {in }}\right)$, causes a lowering of $g^{\prime}(n, E)$. In this case we can still observe dips at the onset of higher order transitions. Since the photon energy $E_{l}$ that maximizes the gain, is near $40-50 \mathrm{meV}$ in both cases of the injected current, this figure indicates that differential gain at $E_{l}$ seems to decrease with the increase of the injected current.

In order to clarify the injected current dependence of the differential gain, we show in Fig. 5 the differential gain $g^{\prime}\left(n, E_{l}\right)$ at maximum gain photon energy $E_{l}$ as a function of the injected current in a MQW laser with various numbers of QW's. The results show that $g^{\prime}\left(n, E_{l}\right)$ has a peaked structure, which is due to the gain saturation effects discussed in Section II. Since $g^{\prime}\left(n, E_{l}\right)$ is a bulk parameter, it does not change with the increase of the number of QW's as long as the Fermi energy is fixed, whereas the injected current increases by a factor of $N$. Consequently, the $g^{\prime}(E, n)$ curve is stretched in the direction of the current axis with an increase of $N$. Note that the second peak appears at $J \sim 300 \mathrm{~mA}$. In the current region below the second peak $(J<300 \mathrm{~mA}), E_{l}$ is located near the first subband. When $E_{l}$ jumps from the first subband to the second subband, this second peak appears.

The differential gain also depends on the QW thickness. Fig. 6 shows the differential gain at $E_{l}$ as a function of the injected current in a SQW laser with the various QW thickness (50 $\AA, 100 \AA$, and $200 \AA$ ). The results indicate two important properties of the $g^{\prime}(n, E)$. First is that the thinner QW laser achieves higher differential gain. This is mainly due to the fact that the contribution of the injected current to the increment of the gain by the next higher subband is enhanced when the QW thickness is larger, and that happens at the expense of gain at $E_{l}$. Sec- 


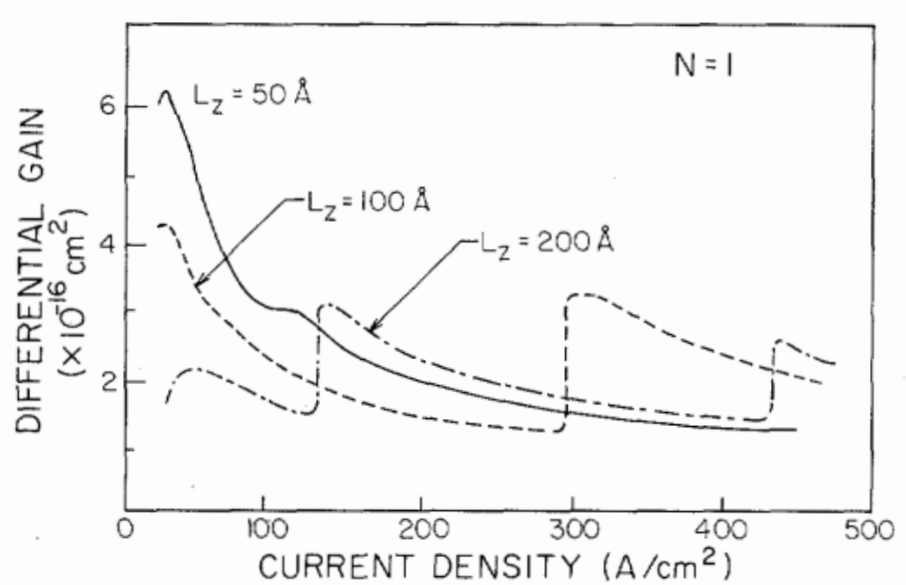

Fig. 6. The differential gain $g^{\prime}\left(n, E_{1}\right)$, at which the gain is maximum, is shown as a function of the injected current with various quantum well thickness $L_{z}$. The number of quantum wells is equal to 1 .

ond is that the $g^{\prime}(n, E)$ has many peaks when $L_{z}=200$ $\AA$. Note that the values of the second and third peak of $g^{\prime}(n, E)$ are comparable to that of the first peak. When $E_{l}$ jumps from the first (second) subband to the second (third) subband, the second peak (third peak) appears. Since thicker QW's have more peaks in the density of states, the jumps appear more frequently with the increase of the $L_{z}$. In the limiting case of thick QW's, $g^{\prime}(n, E)$ increases gradually with the increase of $J$.

\section{B. Modulation Resonance Frequency}

On the basis of the discussion concerning $g^{\prime}(n, E)$ in the previous subsection, we will investigate modulatior dynamics in QW lasers. The rate equation for laser dynamics can be described as follows.

$$
\begin{aligned}
& \frac{d n}{d t}=\frac{J(t)}{e L_{z}}-\frac{n_{r}}{c} g\left(n, E_{1}\right) P-\frac{n}{\tau_{s}} \\
& \frac{d P}{d t}=\Gamma \frac{n_{r}}{c} g\left(n, E_{1}\right) P+\beta \frac{n}{\tau_{s}}-\frac{P}{\tau_{p}}
\end{aligned}
$$

where $P$ is the photon density $\left(\mathrm{cm}^{-3}\right), \beta$ is the spontaneous emission coefficient into the lasing mode, $\tau_{s}$ is the carrier lifetime, $J(t)\left(\mathrm{cm}^{-2}\right)$ is the injected current density, $n$ is the carrier concentration, and $g\left(n, E_{l}\right)\left(\mathrm{cm}^{-1}\right)$ is the bulk gain, while $\Gamma g\left(n, E_{l}\right)$ is the modal gain as a function of the carrier density $n\left(\mathrm{~cm}^{-3}\right)$ at the lasing photon energy $E_{l}$. When we discuss the carrier density in the quantum well structure we usually use the density per $\mathrm{cm}^{2}$, however, the proper "bookkeeping" of photons and carriers requires that $n$ stands for carrier density per unit volume. The relaxation resonance frequency $f_{r}$ is determined by a small-signal analysis of (9). The results can be simply expressed by [1]

$$
f_{r}=\frac{1}{2 \pi} \sqrt{\frac{n_{r} g^{\prime}\left(n, E_{l}\right)}{c} \frac{P_{o}}{\tau_{p}}},
$$

where $P_{o}$ is the photon density in the cavity at stationary states. Note that $\tau_{p}$ is related to the total loss as follows.

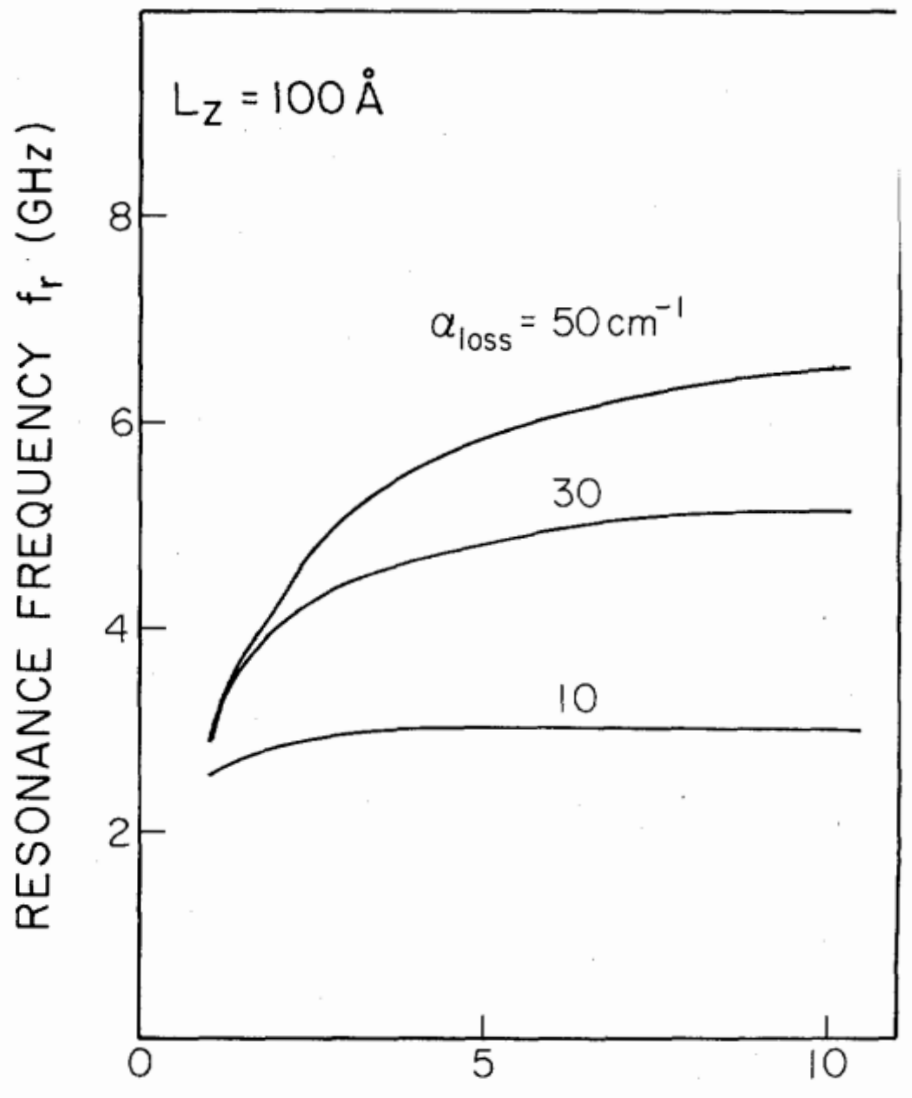

NUMBER OF QUANTUM WELLS

Fig. 7. The calculated results of the resonant frequency $f_{r}$ as a function of $N$ with various total loss $\alpha_{\text {loss }}$, with quantum well thickness $L_{z}=100 \AA$.

$$
\tau_{p}=\frac{n_{r}}{c \alpha_{\text {loss }}} .
$$

It follows from (10) that an increase of the differential gain and of $P_{o}$, together with a decrease of $\tau_{p}$, is required in order to increase the modulation bandwidth. The increase of $P_{o}$ is usually limited by catastrophic mirror damage, while $\tau_{p}$ is determined by the total loss $\alpha_{\text {total }}$. Thus $P_{o}$ and $\tau_{p}$ depend strongly on the laser geometry. On the other hand, the differential gain $g^{\prime}\left(n, E_{l}\right)$ depends on the properties of the QW's and on the injected current at threshold as discussed in connection with Figs. 5 and 6. Fig. 7 shows the calculated result of the resonance frequency $f_{r}$ of MQW lasers with $L_{z}=100 \AA$ as a function of $N$ with $\alpha_{\text {loss }}$ (photon lifetime) as a parameter taking (11) into consideration. As shown in this figure, there is the optimum $N$ which is, usually, not equal to 1 for obtaining high $f_{r}$. This can be explained as follows. When the number of QW's is small, the threshold current $J_{\text {th }}$ is larger than the current $J_{\max }^{N}$ which gives the maximum differential gain because of the gain saturation effects, leading to lower differential gain. On the other hand, the differential gain increases with the increase of $N$, since $J_{\text {th }}$ approaches $J_{\text {max }}^{N}$. However, once $J_{\text {th }}$ becomes smaller than $J_{\max }^{N}$, with the further increase of $N$, the differential gain decreases again. Therefore there exists an optimum number $N_{\text {opt }}$ at which $J_{\max } \sim J_{\text {th }}$. Since $J_{\max }^{N}$ increases with the increase 
of $\alpha_{\text {loss }}$ with the same $N$, the $N_{\text {opt }}$ increases for higher $\alpha_{\text {loss }}$.

In Fig. 8, the maximum resonance frequency $f_{r}$, which can be attained by optimizing $N$ as a function of $L_{z}$ with various $\alpha_{\text {loss }}$, is shown by a solid curve. This result indicates that for the range of losses $\left(\alpha_{\text {loss }}\right)$ considered, the resonance frequency increases with the reduction of the QW thickness: the resonance frequency $f_{r}$ with $L_{z}=50$ $\AA$ is enhanced by 1.8 compared to the $f_{r}$ of the double heterostructure laser.

\section{LASER LINEWIDTH}

\section{A. Spontaneous Emission Factor}

In this section, we analyze the spectral linewidth broadening in the QW lasers and show how a substantial reduction of the linewidth can be obtained with an optimization of the quantum well structures.

The power-dependent linewidth in semiconductor lasers occurs due to the spontaneous emission that modulates the laser field both in intensity and in frequency (phase). This linewidth involves the Schawlow-Townes linewidth that is due to the instantaneous-phase fluctuation as well as to the delayed-phase fluctuation that occurs as the laser intensity returns to its steady states (amplitude phase coupling). The reduced linewidth is [3], [4]

$$
\Delta \nu=\frac{v_{g}^{2} \mathrm{~h} \nu\left(\Gamma g\left(E_{l}\right)\right)\left(L^{-1} \ln (1 / R)\right) n_{\mathrm{sp}}}{8 \pi P_{1}}\left(1+\alpha^{2}\right)
$$

where $P_{1}$ is the output photon density, $\alpha$ is the linewidth enhancement factor, $v_{g}$ is the group velocity of light in the active layer, $h \nu$ is the photon energy, and $n_{\mathrm{sp}}$ is defined as the spontaneous emission rate divided by total stimulated emission rate given by

$$
n_{\mathrm{sp}}(E)=\frac{\int A(\epsilon) \sum_{j=h, l} \sum_{n=1}^{\infty} \rho_{\mathrm{red}_{\mathrm{n}}}^{j}(\epsilon) f_{c}\left(1-f_{v}\right) \frac{\left(\hbar / \tau_{\text {in }}\right)}{(E-\epsilon)^{2}+\left(\hbar / \tau_{\text {in }}\right)^{2}}}{\int A(\epsilon) \sum_{j=h, l} \sum_{n=1}^{\infty} \rho_{\mathrm{red}_{\mathrm{n}}}^{j}(\epsilon)\left(f_{c}-f_{v}\right) \frac{\left(\hbar / \tau_{\text {in }}\right)}{(E-\epsilon)^{2}+\left(\hbar / \tau_{\text {in }}\right)^{2}} d \epsilon} .
$$

If energy broadening is extremely small we can approximate $n_{\mathrm{sp}}$ at the photon energy $E_{l}$ at which the gain is maximum $\left(E=E_{l}\right)$ by

$$
n_{\mathrm{sp}} \approx\left(1-\exp \left(E_{1}-\Delta \epsilon_{F}\right)\right)^{-1}
$$

where $\Delta \epsilon_{F}$ is the difference between the quasi-Fermi energy of electrons and that of holes $\left(\epsilon_{F_{\mathrm{c}}}-\epsilon_{F_{v}}\right)$. Equation (14) shows that $n_{\text {sp }}$ at $E_{l}$ increases substantially with the approach of $\Delta \epsilon_{F}$ to $E_{l}$ (i.e., the total loss decreases), since $E_{l}$ is fixed near $\left(\epsilon_{c 1}-\epsilon_{v 1}\right)$ (the photon energy which corresponds to the lowest subband) in the QW lasers. Finally, $n_{\mathrm{sp}}$ at $E_{l}$ becomes infinite when the injected current is equal to the current $J_{\text {tr }}$ at which transparency occurs $\left(\Delta \epsilon_{F} \rightarrow\right.$ $\left.E_{l}\right)$. In Fig. 9, we show the calculated results of $n_{\mathrm{sp}}$ at $E_{l}$ as a function of the injected current with a various numbers of QW's. The QW thickness is assumed to be $100 \AA$ and $\tau_{\text {in }}$ is assumed to be $0.2 \mathrm{ps}$. The observed dip corresponds to the jump of $E_{l}$ from the electron-hole transition

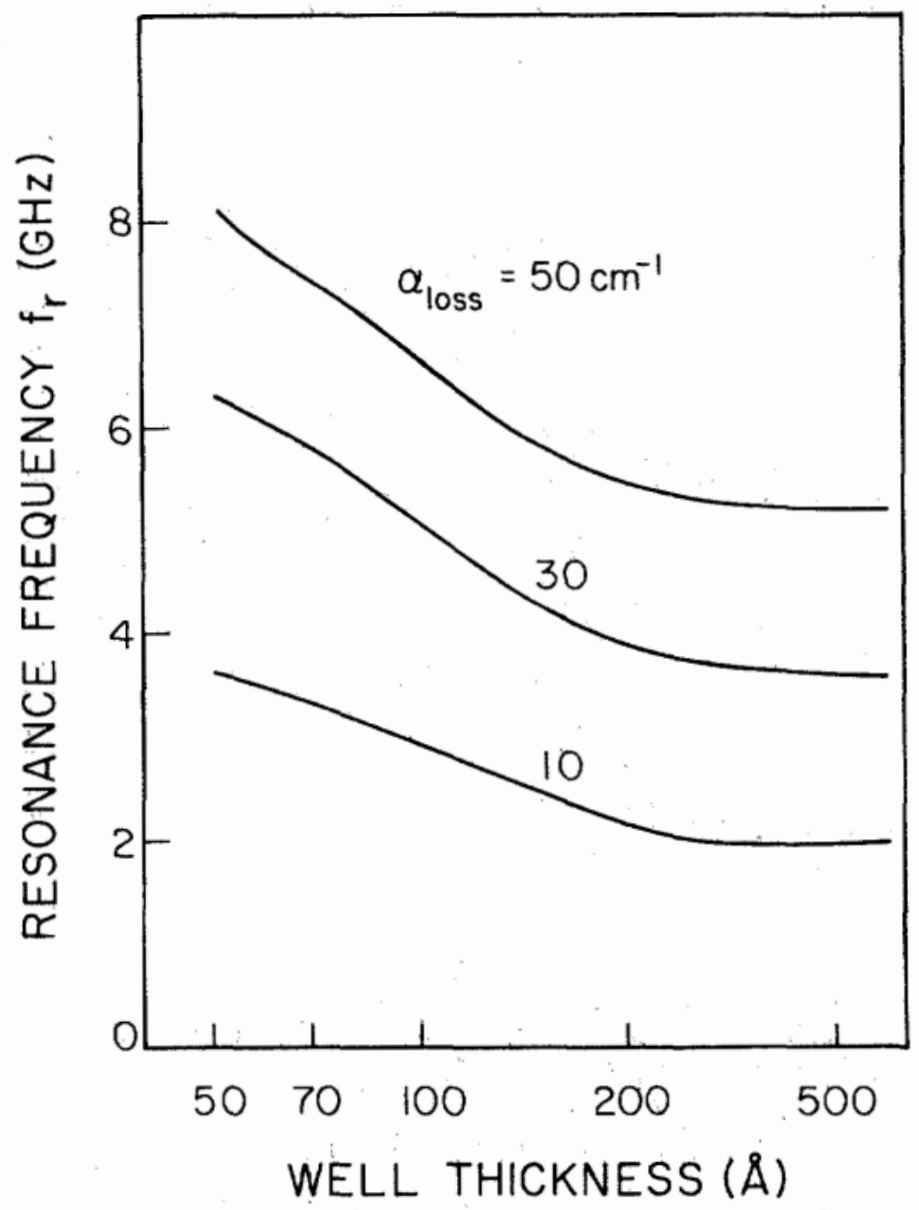

Fig. 8. The maximum resonant frequency, which is achieved by optimizing the number of quantum wells, as a function of the quantum well thickness $L_{z}$ with various total loss $\alpha_{\text {loss }}$. of the first subband to the electron-hole transition of the second subband.

Fig. 10 shows the $n_{\mathrm{sp}}$ at $E_{l}$ as a function of the injected current with various $\mathrm{QW}$ thicknesses. In this calculation, the number of QW's is 1 and $\tau_{\text {in }}=0.2 \mathrm{ps}$. We notice that the approach of $n_{\mathrm{sp}}$ to 1 , with the increase of the injected current, occurs more rapidly when the QW thickness is smaller. We also notice that two dips appear in the case of $L_{z}=200 \AA$. These dips appear when $E_{l}$ jumps from the electron-hole transition of the first (second) subband to the electron-hole transition of the second (third) subband.

\section{B. Linewidth Enhancement Factor}

The linewidth enhancement factor $\alpha$ of (12), which represents the spectral broadening due to the coupling between AM linewidth and FM noise, is given by [17] 


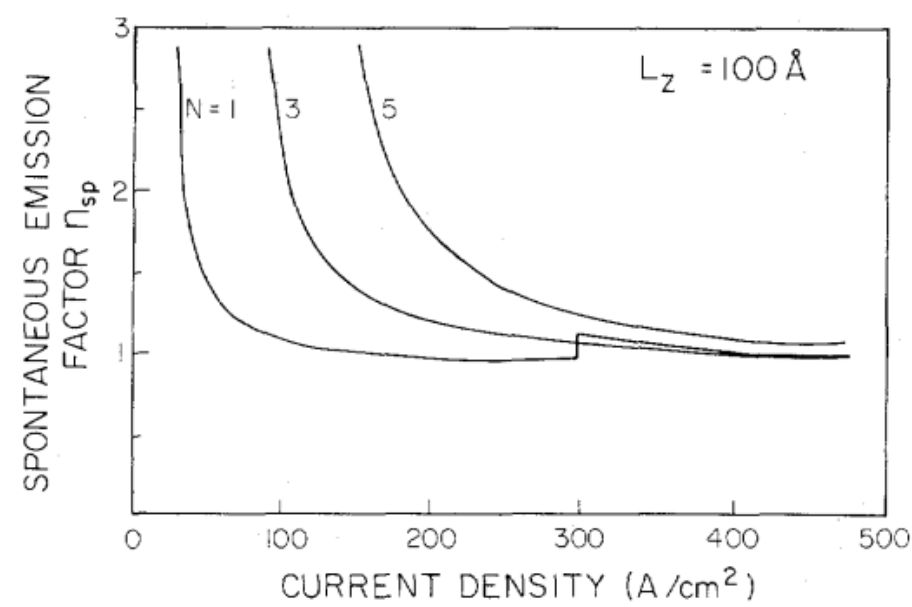

Fig. 9. The spontaneous emission factor $n_{\mathrm{sp}}$ as a function of the injected current, at which the gain is maximum, is shown as a function of the injected current $J$ with various number of quantum wells. The thickness of quantum wells is $100 \AA$.

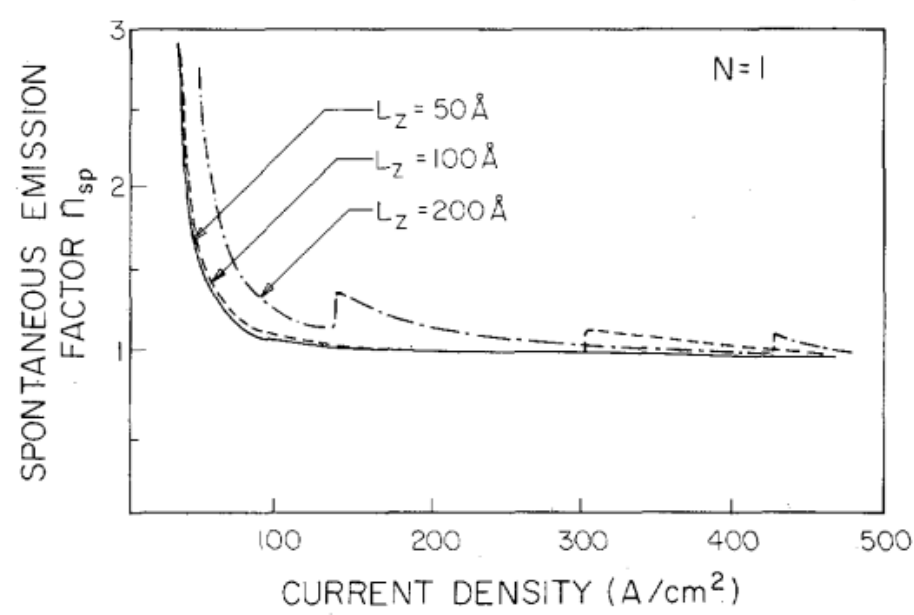

Fig. 10. The spontaneous emission factor $n_{\text {sp }}$ as a function of the injected current, at which the gain is maximum, is shown as a function of the injected current $J$ with various quantum well thickness. The number of quantum wells $N$ is equal to $i$.

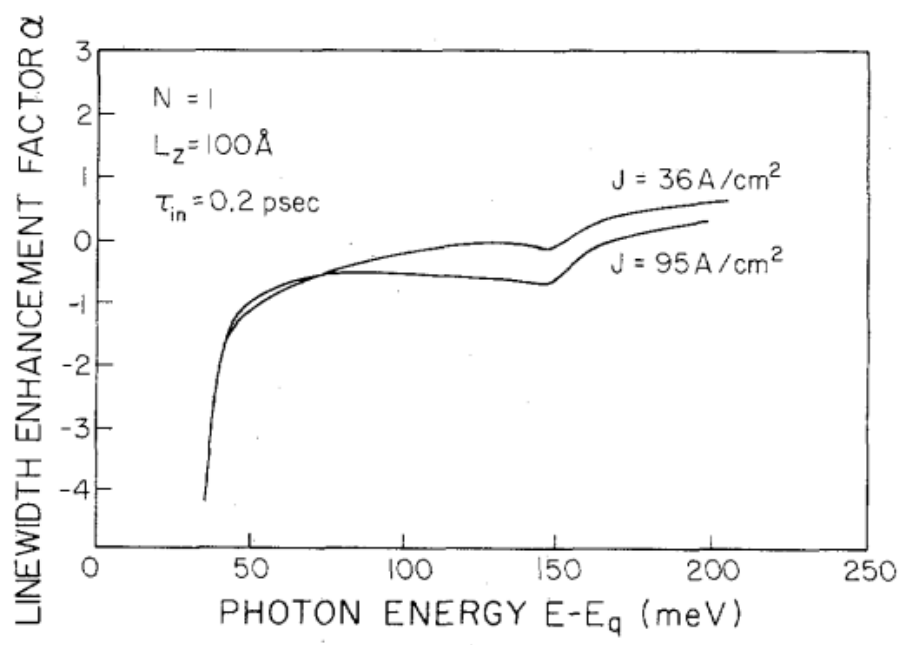

Fig. 11. The linewidth enhancement factor $\alpha$ with various injected currents as a function of the photon energy substracted by the band-gap energy of $\operatorname{GaAs}\left(E-E_{k}\right)$.

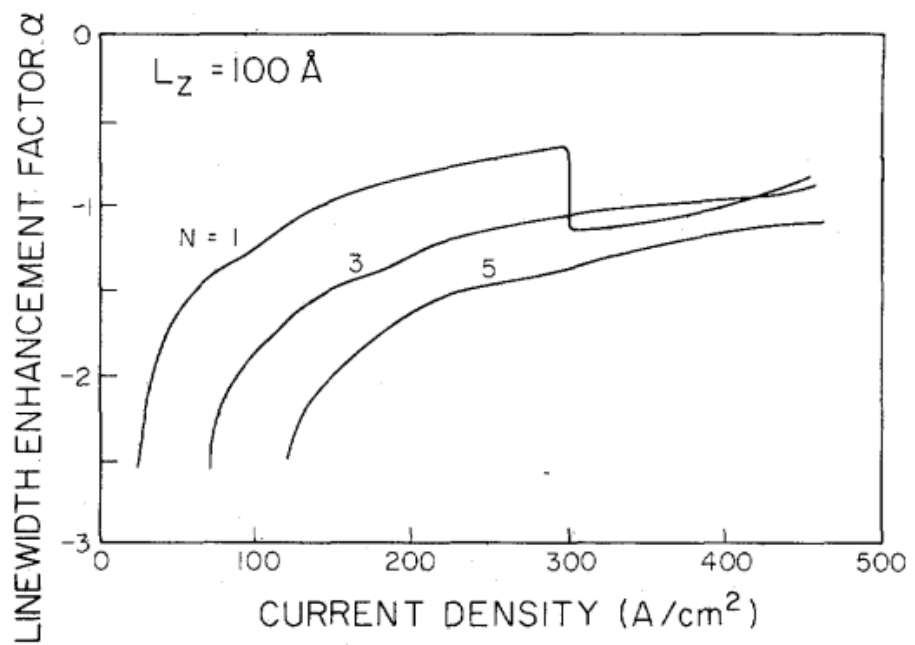

Fig. 12. The linewidth enhancement factor $\alpha$ as a function of the injected current at photon energy, at which the gain is maximum with various $N$. The thickness of quantum wells is $100 \AA$.

$$
\alpha=\frac{\int A(\epsilon) \sum_{j=j h, l} \sum_{n=1}^{\infty} \rho_{\mathrm{red}_{n}}^{j}(\epsilon)\left(\frac{d f_{c}\left(\epsilon_{c_{n}}\right)}{d n}-\frac{d f_{v}\left(\epsilon_{v_{n}}^{j}\right)}{d n}\right) \frac{(E-\epsilon)}{(E-\epsilon)^{2}+\left(\hbar / \tau_{\text {in }}\right)^{2}} d \epsilon}{\int A(\epsilon) \sum_{j=h, l} \sum_{n=1}^{\infty} \rho_{\mathrm{red}_{n}}^{j}(\epsilon)\left(\frac{d f_{c}\left(\epsilon_{c_{n}}\right)}{d n}-\frac{d f_{v}\left(\epsilon_{v_{n}}^{j}\right)}{d n}\right) \frac{\left(\hbar / \tau_{\text {in }}\right)}{(E-\epsilon)^{2}+\left(\hbar / \tau_{\text {in }}\right)^{2}} d \epsilon} .
$$

Fig. 11 shows the $\alpha$ parameter as a function of $\left(E-E_{g}\right)$ (the photon energy minus the band-gap energy) for injected currents of $J=36 \mathrm{~A} / \mathrm{cm}^{-2}$, and $J=95 \mathrm{~A} / \mathrm{cm}^{-2}$, respectively. We notice that $\propto$ increases with the increase of the photon energy and also changes its sign from minus to plus. This is due to the change in the sign of the numerator of (15) from minus to plus, since the differential gain profile has a peaked structure as indicated in Fig. 4. Fig. 12 shows $\alpha$ at the maximum gain photon energy $E_{l}$ as a function of the injected current for various $N$ 's. In this calculation the QW thickness and $\tau_{\text {in }}$ are assumed to be $100 \AA$, and 0.2 ps, respectively. Since $\alpha$ is a bulk parameter, the value of $\alpha$ is independent of the number of quantum wells $N$, so that the injected current to achieve a given value of $\alpha$ is proportional to $N$, and the currents for
$N=1,3,5$ differ only in their horizontal scale. We find that the absolute value of $\alpha(|\alpha|)$ decreases with the increases of the injected current. Therefore we can reduce the value of $|\alpha|$ by the use of high loss $\alpha_{\text {loss }}$ although this leads to high-threshold current.

Fig. 13 shows the $\alpha$ parameter as a function of the injected current with various $\mathrm{QW}$ thickness at $E_{l}$. The value of $N$ is taken as unity. We notice a tendency that the smaller $L_{\mathrm{z}}$ is, the smaller $\alpha$ is.

\section{Spectral Linewidth}

Fig. 14 shows the laser spectral width $\Delta \nu$ calculated from (11) as a function of the number of QW's with various total loss $\left(\alpha_{\text {total }}=10,30,50 \mathrm{~cm}^{-1}\right)$. We find that the 


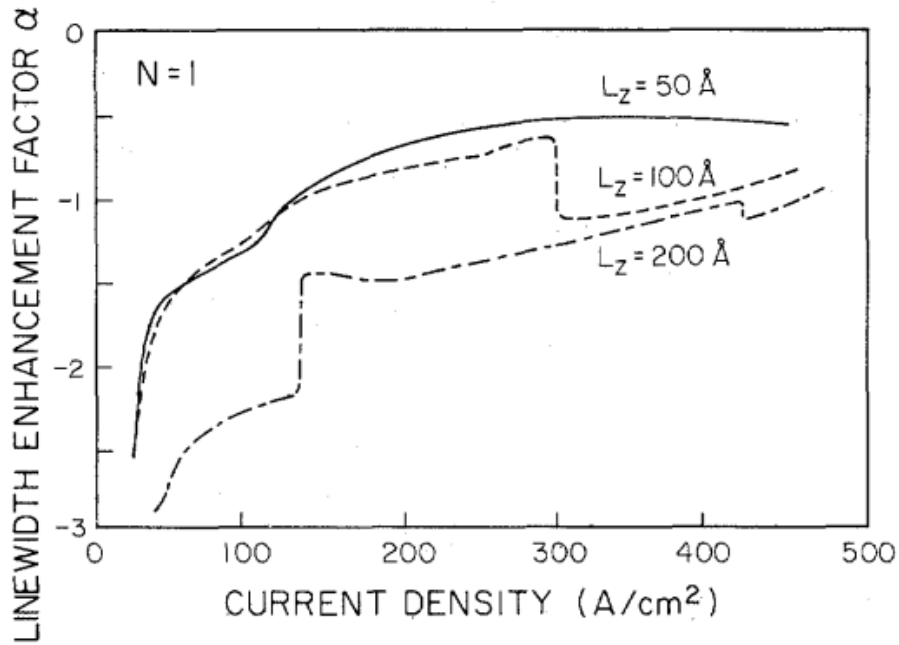

Fig. 13. The linewidth enhancement factor $\alpha$ as a function of the injected current with various quantum well thickness. The thickness of quantum wells is $100 \AA$.

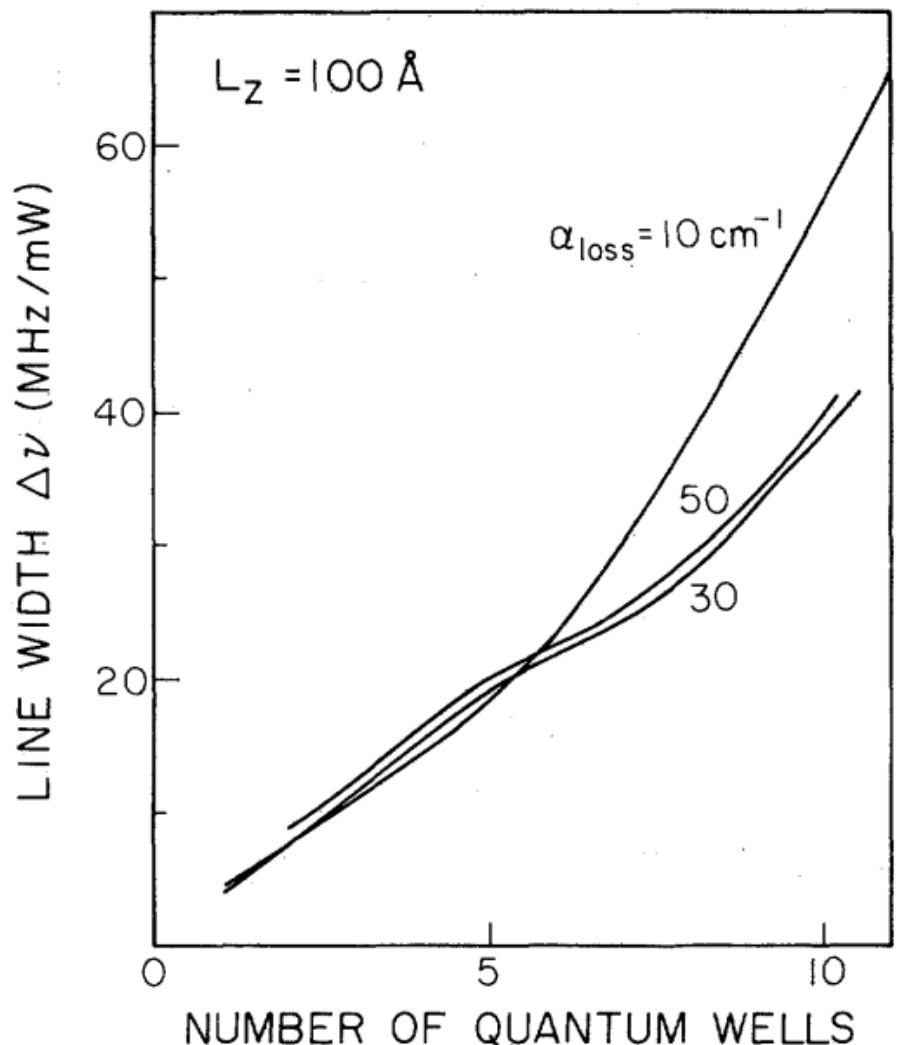

Fig. 14. The spectral linewidth $\Delta v$ as a function of the number of quantum wells with various total loss $\alpha_{\text {loss }}$.

$\Delta \nu$ increases monotonically with the increase of the number of QW's. This is due to the fact that both $\propto$ and $n_{\text {sp }}$ are reduced with the increase of $\Delta \epsilon_{F}$ which is enhanced with small number of $N$. Therefore, in order to reduce $\Delta \nu$, a SQW laser should be used rather than a MQW laser. The substantial change in $\Delta \nu$ with the increase of $N$ is due to the fact that the abrupt change in $\alpha$ occurs in the low injected-current region. Note that this change is enhanced with low $\alpha_{\text {loss }}$.

The spectral width $\Delta \nu$ is also a function of the thickness $L_{z}$. Fig. 15 shows the minimum attainable $\Delta \nu$ as a function

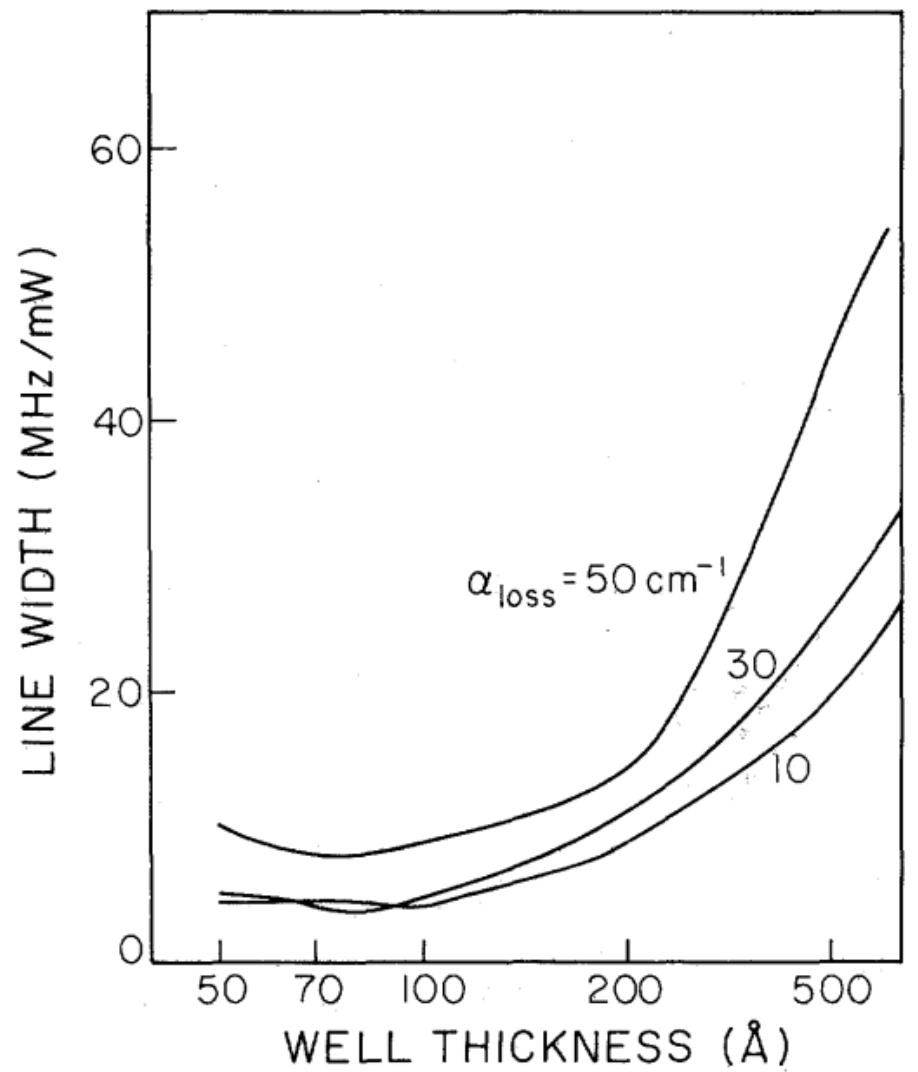

Fig. 15. The minimum spectral linewidth $\Delta \nu$ as a function of the quantum well thickness with various total loss. The number of quantum wells is optimized.

of the QW thickness with various $\alpha_{\text {loss }}$. Generally speaking, we notice that $\Delta \nu$ is reduced greatly in the region of thin active layer. In the case of $\alpha_{\text {loss }}=10 \mathrm{~cm}^{-1}, \Delta \nu$ is minimum arround $L_{z}=80 \AA$. This is due to the fact that there is the current region in which $\propto$ of $L_{z}=100 \AA$ is smaller than that of $L_{z}=50 \AA$, as shown in Fig. 13. Since the value $\Delta \nu$ for a DH laser $(0.1 \mu \mathrm{m}$ active layer $)$ is calculated to be $60 \mathrm{MHz} / \mathrm{mW}$ with $\alpha_{\text {loss }}=30 \mathrm{~cm}^{-1}$, the $\Delta \nu$ can be substantially reduced with a thin QW structure by factor of $\sim 1 / 10$ compared to DH lasers. For all $L_{z}$, the $\Delta \nu$ increases monotonically when the number of QW's increases.

\section{Conclusions}

We investigate theoretically the effects of quantum well (QW) structure parameters such as the number and the thickness of QW's on the gain, the dynamic response and quantum noise of QW lasers. We have considered the issues of optimum conditions for realizing low-noise characteristics and high-dynamic performance. We have found that the gain in structures with a small number of layers tends to saturate with current. The differential gain and linewidth enhancement factor of QW lasers are also discussed. The results indicate that the modulation bandwidth can be enhanced by a factor of $\sim 2$ in the multiquantum well laser, and linewidth is found to be significantly suppressed by $1 / 10$ in a single thin QW laser compared to the conventional double heterostructure laser. 


\section{REFERENCES}

[1] K. Y. Lau, N. Bar Chaim, I. Ury, Ch. Harder, and Y. Yariv, "Direct amplitude modulation of short-cavity GaAs lasers up to X-band frequency," Appl. Phys. Lett., vol. 43, pp. 1-3, 1983.

[2] M. W. Fleming and A. Mooradian, "Fundamental line broadening of single mode (GaAl)As diode lasers," Appl. Phys. Lett., vol. 38, pp. $511-513,1981$.

[3] C. Henry, "Theory of the linewidth of semiconductor lasers," IEEE J. Quantum Electron., vol. QE-18, pp. 259-254, 1982.

[4] K. Vahala and A'. Yariv, "Semiclassical theory of noise in semiconductor lasers-Part 1," IEEE J. Quantum Electron., vol. QE-18, pp. 1096-1101, 1982.

[5] K. Vahala and A. Yariv, "Semiclassical theory of noise in semiconductor lasers-Part 2," IEEE J. Quantum Electron., vol. QE-18, pp. $1102-1109,1982$.

[6] K. Vahala and A. Yariv, "Detuned loading in coupled cavity semiconductor lasers-Effects on quantum noise and dynamics," Appl. Phys. Lett., vol. 45, pp, 501-503, 1984.

[7] N. Holonyak, Jr., R. M. Kolbas, R. D. Dupuis, and P. D. Dapkus, "Quantum-well heterostructure lasers," IEEE J. Quantum Electron., vol. QE-16, p. 170, 1980.

[8] R. D. Dupuis, P. D. Dapkus, N. Holonyak, Jr., E. A. Rezek, and R. Chin, "Room-temperature laser operation of quantum-well $\mathrm{Ga}_{1-x} \mathrm{Al}_{x} \mathrm{As}-\mathrm{GaAs}$ laser diodes by metalorganic chemical vapor deposition," Appl. Phys. Lett., vol. 32, pp. 295-297, 1978.

[9] W. T. Tsang, C. Weisbuch, R. C. Miller, and R. Dingle, "Current injection $\mathrm{GaAs}-\mathrm{Al}_{x} \mathrm{Ga}_{1}{ }_{x} \mathrm{As}$ multiquantum well heterostructure lasers prepared by molecular beam epitaxy," Appl. Phys. Lett., vol. 35, pp. $673-675,1979$.

[10] W. T. Tsang, "A graded-index waveguide separate-confinement laser with very low threshold and a narrow Gaussian beam," Appl. Phys. Lett., vol. 39, pp. 134-136, 1981.

[11] W. T. Tsang, "Extremely low threshold (AlGa)As modified multiquantum well heterostructure lasers grown by molecular beam epitaxy," Appl. Phys. Lett., vol. 39. pp. 786-788, 1981.

[12] Y. Arakawa, K. Vahala, and A. Yariv, "Quantum noise and dynamics in quantum well and quantumwire lasers," Appl. Phýs. Lett., vol. 45, pp. $950-952,1984$.

[13] M. G. Burt, "Linewidth enhancement factor for quantum well lasers," Electron. Lett., vol. 20, pp. 27-28, 1984.

[14] Kobayashi, H. Iwamura, T. Saku, K. Otsuka, and H. Okamoto, "Dynamic behavior of a GaAs-AlGaAs MQW laser diode," Electron. Lett., vol. 19, pp. 166-167, 1983.
[15] A. Sugimura, "Threshold current for AlGaAs quantum well lasers," IEEE J. Quantum Electron., vol. QE-20, no. 4, pp. 336-343, 1984.

[16] M. Asada, A. Kameyama, and Y. Suematsu, "Gain and intervalence band absorption in quantum-well lasers," IEEE J. Quantum Electron., vol. QE-20, no. 7, pp. 745-753, 1984.

[17] K. Vahala, L. C. Chiu, S. Margalit, and A. Yariv, "On the linewidth enhancement factor $\alpha$ in semiconductor injection lasers," Appl. Phys. Lett., vol. 42, pp. 631-633, 1983.

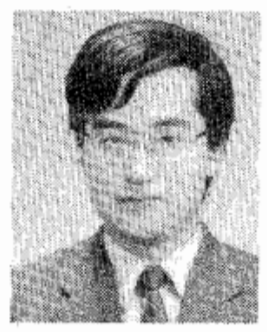

Yasuhiko Arakawa $\left(\mathrm{S}^{\prime} 77-\mathrm{M}^{\prime} 80\right)$ was born in $\mathrm{Ai}-$ chi Prefecture, Japan, on November 26; 1952. He received the B.S., M.S., and Ph.D. degrees in electrical engineering from the University of Tokyo, in 1975,1977 , and 1980 , respectively. In the graduate school, he conducted research on optical communication theory.

In 1980, he joined the Institute of Industrial Science, University of Tokyo, as an Assistant Professor and is now an Associate Professor. After 1980 , he extended his speciality to optical device research. His current research includes dynamic and noise properties of quantum well lasers, basic investigation of quantum wire and quantum box lasers using high magnetic fields, and fabrication of new optical devices using quantum well structures. From 1984 to 1986, he will be a Visiting Scientist at the California Institute of Technology, Pasadena, doing research in collaboration with Professor A. Yariv.

Dr. Arakawa is a member of the Institute of Electronics and Communication Engineers of Japan and Japan Society of Applied Physics. In 1981, he was awarded the Excellent Paper Award for "the proposal of a new type transmission code for digital optical communication" from Institute of Electronics and Communication Enginners of Japan. For this work, he was also awarded Niwa Memorial Prize in 1980. In 1983, he was awarded the Young Scientist Award for "study on the light emission from one-dimensional electronic systems" from the Institute of Electronics and Communication Engineers of Japan.

Amnon Yariv, (S'56-M'59-F'70), for a photograph and biography, see p. 138 of the February 1985 issue of this JoURNAL. 